\title{
Reliability improvement and risk reduction by inequalities and segmentation
}

\author{
Michael Todinov \\ School of Engineering, Computing and Mathematics \\ Oxford Brookes University, Oxford, UK \\ mtodinov@brookes.ac.uk
}

\begin{abstract}
The paper introduces new domain-independent methods for improving reliability and reducing risk based on algebraic inequalities and chain-rule segmentation.

Two major advantages of algebraic inequalities for reducing risk have been demonstrated: (i) ranking risky prospects in the absence of any knowledge related to the individual building parts and (ii) reducing the variability of a risk-critical critical output parameter. The paper demonstrates a highly counter-intuitive result derived by using inequalities: if no information about the component reliability characterising the individual suppliers is available, purchasing components from a single supplier or from the smallest possible number of suppliers maximises the probability of a high-reliability assembly.

The paper also demonstrates the benefits from combining domain-independent methods and domain-specific knowledge for achieving risk reduction in several unrelated domains: decision-making, manufacturing, strength of components and kinematic analysis of complex mechanisms. In this respect, the paper introduces the chain rule segmentation method and applies it to reduce the risk of computational errors in kinematic analysis of complex mechanisms. The paper also demonstrates that combining the domain-independent method of segmentation and domain-specific knowledge in stress analysis leads to a significant reduction of the internal stresses and reduction of the risk of overstress failure.
\end{abstract}

Keywords: risk reduction; reliability improvement; domain-independent methods; inequalities, segmentation

\section{Introduction}

For many decades, the focus of the risk research has been exclusively on identifying risks, risk assessment and risk management rather than general methods for reliability improvement and risk reduction. While a great deal of agreement exists about the necessary common steps of risk assessment (Aven, 2016) and they can be considered to be domain-independent, there is insufficient research on general methods for reducing risk that work in various unrelated domains.

There is a strong perception that effective risk reduction can be delivered solely by using methods offered by the specific domains, without resorting to a general risk reduction 
methodology. As a result, in textbooks on mechanical engineering and design of machine components, for example, (Budynas and Nisbett 2015; Childs, 2014; Thompson, 1999; French, 1999; Samuel and Weir, 1999; Collins, 2003; Norton, 2006; Pahl and Beitz, 2007), there is no reference to general methods for improving reliability and reducing the risk of failure of mechanical components.

Even in standard reliability textbooks (e.g. Lewis,1996; Ebeling, 1997; O'Connor 2002; Dhillon 2017; Modarres et al, 2017) there is a surprising lack of discussion related to domainindependent methods for improving reliability and reducing risk. The discussion is limited to few popular domain-independent methods for risk reduction such as: implementing redundancy, derating, eliminating a common cause, reducing variability, robust design, simplification and condition monitoring.

Recently, a number of new domain-independent methods and principles for improving reliability and reducing risk have been presented in (Todinov, 2019). The objective of the present paper is to extend the work on new domain-independent methods for risk reduction. This has been done by introducing new domain-independent methods for improving reliability and reducing risk based on algebraic inequalities and chain-rule segmentation.

Strength of components and kinematic analysis of complex mechanisms are mature and well-developed fields (Hearn 1985; Budynas, 1999; Gere and Timoshenko, 1999; Budynas and Nisbett 2015; Collins 2003; Norton, 2006; Uicker et al, 2017; Dicker et al, 2003; Sandor and Erdman, 1984). Despite this, to the best of our knowledge, nowhere in standard textbooks related to these fields, have the ideas of segmenting external loads and chain rule segmentation been used to reduce risk. In this respect, the paper demonstrates the benefits from combining domain-independent methods and domain-specific knowledge for achieving risk reduction in mature areas such as strength of components and kinematic analysis of complex mechanisms.

By using inequalities, the paper also demonstrates the significant benefits from combining domain-independent methods and domain-specific knowledge for risk reduction in decisionmaking and manufacturing. In these specific domains, the paper demonstrates the big potential of non-trivial algebraic inequalities in ranking risky prospects in complete absence of knowledge related to key parameters. There are a number of useful non-trivial algebraic inequalities such as the Arithmetic mean - Geometric mean (AM-GM) inequality, CauchySchwartz inequality, the rearrangement inequality, the Chebyshev's inequality, Jensen's inequality, Muirhead's inequality, etc. Non-trivial algebraic inequalities have been discussed extensively in (Steele, 2004; Cloud et al. 1998; Engel 1998; Hardy et al., 1999; Kazarinoff, 
1961; Pachpatte 2005). In probability theory, well-known non-trivial inequalities are the Tchebyshev's inequality, Markov's inequality, Boole's inequality, Bonferroni inequalities and Jensen's inequality. Some of these inequalities have been used in physics (Rastegin, 2012) and reliability theory (Ebeling, 1997) for obtaining lower and upper bound on the system reliability by using minimal cut sets and minimal path sets.

Despite the existence of a well-developed theory of non-trivial algebraic inequalities, there is a clear lack of discussion related to their application to reducing risk. In engineering design, the application is mainly confined to trivial inequalities. Design variables are required to satisfy various trivial design inequalities in order to guarantee that a number of failure modes will be eliminated and the design will perform its required functions (Samuel and Weir, 1999). Trivial inequality constraints have, for example, been introduced in (Ning-Cong et al., 2013) for describing the dependency of interval variables into a non-probabilistic model. Trivial inequalities, obtained by solving with respect to one of the variables have been used for specifying the upper bound of the lineal density of Poisson-distributed flaws to guarantee a probability of clustering below a maximum acceptable level (Todinov 2005).

Why are inequalities important for reliability improvement and risk reduction?

While the equalities express a state of equivalence, equilibrium and absence of transition, inequalities express ranking for the compared alternatives. In addition, inequalities do not normally require any knowledge related to the values of the controlling variables.

Suppose that two different system configurations are built by using the same set of $n$ components with performance characterisics (e.g. reliabilities) $x_{1}, x_{2}, \ldots, x_{n}$ that are unknown. Let the performance of the first configuration be given by the function $f\left(x_{1}, \ldots, x_{n}\right)$ while the performance of the second configuration be given by $g\left(x_{1}, \ldots, x_{n}\right)$. If an equality of the type

$$
f\left(x_{1}, \ldots, x_{n}\right)>g\left(x_{1}, \ldots, x_{n}\right)
$$

could be proved, this would mean that the performance (e.g. reliability) of the first configuration is superior to the performance of the second configuration. Then the first system configuration can be selected and the risk of failure reduced in the absence of any knowledge related to the reliabilities of the parts building the systems. The possibility of making a correct ranking of two competing systems/processes under a complete absence of knowledge about the reliabilities of their building parts, constitutes a formidable advantage of algebraic inequalities. This advantage was demonstrated in Todinov (2019), where inequalities, proved by a direct algebraic manipulation, have been used for ranking the reliabilities of systems in the case where the reliabilities of their components are unknown. 
This advantage will also be demonstrated in this paper with the application of the Muirhead's inequality for ranking risky prospects.

The next major advantage of the non-trivial algebraic inequalities as a domainindependent method for reducing risk can be found in their capacity to produce sharp bounds related to uncertainty associated with reliability-critical design parameters (e.g. material properties, dimensions, loads). In many cases, the actual values of the reliability-critical parameters are unknown or are associated with large variability. If a bound can be determined related to existing epistemic or aleatoric uncertainty, the design could be complied with this worst possible bound and a number of failure modes could be avoided.

Such a case is present for mechanical properties from multiple sources where the proportions with which the sources are present in the common pool of properties are unknown. Determining a sharp upper bound for the variation of properties helps to improve the robustness of the design. Consequently, inequalities producing such sharp bounds could yield reliability improvement and risk reduction.

Another major advantage of algebraic inequalities is that they work well in limiting the uncertainty associated with the variation of a critical output parameter and this application will be demonstrated in the manuscript.

\section{Using the Muirhead's inequality for improving reliability and reducing risk}

Consider a real-world example where three suppliers $A_{1}, A_{2}$ and $A_{3}$, produce high-reliability components of the same type, with probabilities $x_{1}, x_{2}$ and $x_{3}$, which are unknown. Probability $x_{i}$ means that only a fraction $x_{i}$ of the components produced by supplier $i$ are of high-reliabiliy and the rest are not. In the case of suspension automotive springs for example, this means that only a fraction $x_{i}$ of the manufactured suspension springs can last for more than 600000 cycles if tested on a specially designed test rig and the rest of the springs fail significantly below this limit.

If two components are to be purchased and installed in an assembly, the question of interest is: which strategy maximises the probability that both components will be highly reliable?: (i) purchasing the two components from the same supplier or (ii) purchasing the two components from different suppliers. At a first glance, it seems that either of these 
strategies could be chosen because the probabilities $x_{1}, x_{2}$ and $x_{3}$ of high-reliability components characterising the suppliers are unknown. Surprisingly, this common-sense conclusion is incorrect.

The probability of purchasing two high-reliability components from the same supplier is: $p_{1}=(1 / 3) x_{1}^{2}+(1 / 3) x_{2}^{2}+(1 / 3) x_{3}^{2}$ and is composed of the probabilities of three mutually exclusive events: (i) the probability $(1 / 3) x_{1}^{2}$ that supplier $A_{1}$ will be selected and both components purchased from $A_{1}$ will be highly reliable; (ii) the probability $(1 / 3) x_{2}^{2}$ that supplier $A_{2}$ will be selected and both components purchased from $A_{2}$ will be highly reliable and (iii) the probability $(1 / 3) x_{3}^{2}$ that supplier $A_{3}$ will be selected and both components purchased from $A_{3}$ will be highly reliable.

Accordingly, the probability of purchasing two high-reliability components from two different suppliers is $p_{2}=(1 / 3) x_{1} x_{2}+(1 / 3) x_{1} x_{3}+(1 / 3) x_{2} x_{3}$.

The probability is composed of the probabilities of three mutually exclusive events: (i) the probability

$$
(1 / 3) \times(1 / 2) x_{1} x_{2}+(1 / 3) \times(1 / 2) x_{2} x_{1}=(1 / 3) x_{1} x_{2}
$$

that suppliers $A_{1}$ and $A_{2}$ will be randomly selected and both components purchased from $A_{1}$ and $A_{2}$ will be of high reliability; (ii) the probability (1/3) $x_{1} x_{3}$ that suppliers $A_{1}$ and $A_{3}$ will be randomly selected and both components purchased from $A_{1}$ and $A_{3}$ will be of high reliability and (iii) the probability $(1 / 3) x_{2} x_{3}$ that suppliers $A_{2}$ and $A_{3}$ will be randomly selected and both components purchased from $A_{2}$ and $A_{3}$ will be of high reliability.

The question is reduced to comparing the probabilities $p_{1}$ and $p_{2}$. Consequently, the problem is reduced to proving (or disproving) the inequality $p_{1}>p_{2}$

The last inequality follows from the general Muirhead's inequality (2), which is discussed next.

Muirhead's inequality: If the sequence $\{a\}$ majorizes the sequence $\{b\}$ and $x_{1}, x_{2}, \ldots, x_{n}$ are non-negative, the inequality

$$
\sum_{s y m} x_{1}^{a_{1}} x_{2}^{a_{2}} \ldots x_{n}^{a_{n}} \geq \sum_{s y m} x_{1}^{b_{1}} x_{2}^{b_{2}} \ldots x_{n}^{b_{n}}
$$

holds (Hardy et al, 1999). 
Consider the two non-increasing sequences $a_{1} \geq a_{2}, \ldots, \ldots a_{n}$ and $b_{1} \geq b_{2} \geq, \ldots, \geq b_{n}$ of positive real numbers. The sequence $\{a\}$ is said to majorize the sequence $\{b\}$ if the following conditions are fulfilled:

$$
\begin{array}{r}
a_{1} \geq b_{1} ; a_{1}+a_{2} \geq b_{1}+b_{2} ; \ldots ; a_{1}+a_{2}+\ldots+a_{n-1} \geq b_{1}+b_{2}+\ldots+b_{n-1} ; \\
a_{1}+a_{2}+\ldots+a_{n-1}+a_{n}=b_{1}+b_{2}+\ldots+b_{n-1}+b_{n}
\end{array}
$$

For any set of non-negative numbers $x_{1}, x_{2}, \ldots, x_{n}$, a symmetric sum is defined as $\sum_{\text {sym }} x_{1}^{a_{1}} x_{2}^{a_{2}} \ldots x_{n}^{a_{n}}$ which, when expanded, includes $n$ ! terms. Each term is formed by a distinct permutation of the elements of the sequence $a_{1}, a_{2}, \ldots, a_{n}$. Thus, if $\{a\}=[2,1,0]$ then $\sum_{s y m} x_{1}^{2} x_{2}^{1} x_{3}^{0}=x_{1}^{2} x_{2}+x_{1}^{2} x_{3}+x_{2}^{2} x_{1}+x_{2}^{2} x_{3}+x_{3}^{2} x_{1}+x_{3}^{2} x_{2}$ If $\{a\}=[2,0,0]$, then $\sum_{s y m} x_{1}^{2} x_{2}^{0} x_{3}^{0}=2 x_{1}^{2}+2 x_{2}^{2}+2 x_{3}^{2}$

Consider now the set of non-negative numbers $x_{1}, x_{2}, x_{3}$ and the sequences $\{a\}=[2,0,0]$ and $\{b\}=[1,1,0]$. Clearly, the sequence $\{a\}=[2,0,0]$ majorizes the sequence $\{b\}=[1,1,0]$ because the conditions (3) are fulfilled:

$2 \geq 1 ; 2+0 \geq 1+1$ and $2+0+0 \geq 1+1+0$.

According to the Muirhead's inequality (2):

$$
2 ! \times\left(x_{1}^{2}+x_{2}^{2}+x_{3}^{2}\right) \geq 2\left(x_{1} x_{2}+x_{1} x_{3}+x_{2} x_{3}\right)
$$

Dividing both sides of the last inequality by the positive constant 3 ! leads to

$$
\left.p_{1}=(1 / 3) x_{1}^{2}+(1 / 3) x_{2}^{2}+(1 / 3) x_{3}^{2} \geq p_{2}=(1 / 3) x_{1} x_{2}+(1 / 3) x_{1} x_{3}+1 / 3\right) x_{2} x_{3}
$$

According to inequality (4), $p_{1} \geq p_{2}$ therefore purchasing both components from a single, randomly selected supplier is the better strategy, resulting in a higher probability that both components will be high-reliability components. This is a surprising and highly counterintuitive result. After all, the percentages of high-reliability components characterising the suppliers are unknown.

Unexpected as it may seem, the conclusion has been confirmed by a Monte Carlo simulation. Consider three suppliers $A, B$ and $C$, characterised by probabilities of high-reliability components $a_{1}=0.9, a_{2}=0.4$ and $a_{3}=0.3$. The Monte Carlo simulation based on one million trials yields 0.35 for the probability of two high-reliability components if a single 
supplier is randomly selected and 0.25 for the probability of two high-reliability components if two suppliers are randomly selected. These values coincide with the values evaluated from the left and right hand side of (4).

The basic idea behind the simulation of purchasing two components from a randomly selected supplier and testing the components for high-reliability is done within a loop of ten million trials. The pseudo-code fragment is shown next:

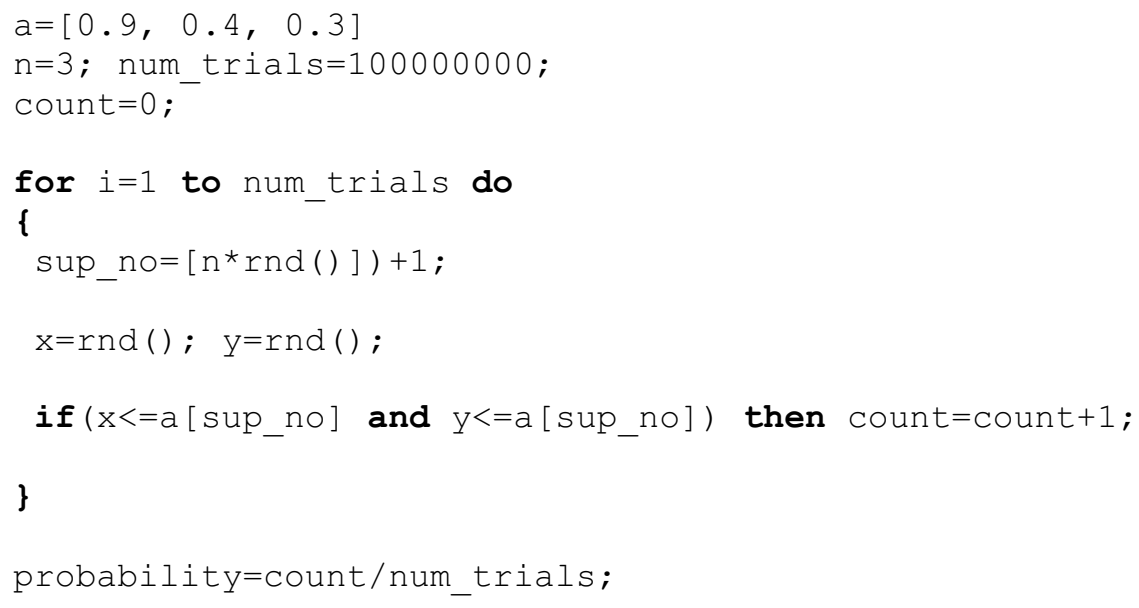

A supplier is selected randomly with the statement $\left.\sup _{-}{ }=\left[n^{\star} r n d\right]\right)+1$; where $[n * r n d]$ is the greatest integer part that does not exceed $n *$ rnd () and $r n d()$ is a function that returns a random number from the interval $(0,1)$. The probabilities of a high-reliability component are specified in the array a[]. In the test case, $a[0.9,0.4,0.3]$ has been specified for the three suppliers. The test for two high-reliability components is performed by the conditional statement

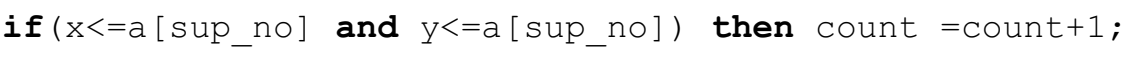

where $x$ and $y$ are two random numbers from the interval $(0,1)$. If the compound condition $\mathrm{x}<=a\left[\sup _{-} \mathrm{no}\right]$ and $\mathrm{y}<=\mathrm{a}\left[\sup _{-} \mathrm{no}\right]$ is true, this means that both components are highreliability components in which case the counter count of two high-reliability components is incremented. If the compound condition is false, the counter count is not incremented. Finally, the probability of purchasing two high-reliability components from a randomly selected supplier is obtained by dividing the content of the counter to the number of trials.

Simulating purchasing of two high-reliability components from two randomly selected suppliers is done in a similar fashion and details will be omitted. First, a supplier is randomly selected, then from the remaining two suppliers one more supplier is randomly selected.

The same technique based on reducing to the Muirhead's general inequality can be extended for $n$ different suppliers. 
The probability of purchasing two high-reliability components from a randomly selected supplier is $p_{1}=\frac{1}{n} \sum_{i=1}^{n} x_{i}^{2}$. Accordingly, the probability of purchasing two high-reliability components from two randomly selected suppliers is $p_{2}=\frac{2}{n(n-1)} \sum_{i<j} x_{i} x_{j}$.

Since the sequence $\{a\}=[2,0,0, \ldots, 0]$ (containing $n$ elements) majorizes the sequence $\{b\}=[1,1,0, \ldots, 0]$ (also containing $n$ elements), according to the Muirhead's inequality (2):

$$
(n-1) ! \times \sum_{i=1}^{n} x_{i}^{2} \geq(n-2) ! \times 2 \times \sum_{i<j} x_{i} x_{j}
$$

Dividing both sides of the last inequality by the positive number $n$ ! yields

$$
p_{1}=\frac{1}{n} \sum_{i=1}^{n} x_{i}^{2} \geq p_{2}=\frac{2}{n(n-1)} \sum_{i<j} x_{i} x_{j}
$$

The left hand side of (5) is the probability of purchasing two high-reliability components from a randomly selected single supplier while the right hand side of (5) is the probability of purchasing two high-reliability components from two distinct, randomly selected suppliers.

Muirhead's inequality can also be applied for a larger number of purchased components. If, for example, three components are to be purchased from three suppliers $(n=3)$ and installed in an assembly, the question of interest is to choose between several competing strategies: a) purchasing the three components from a single, randomly selected supplier; b) purchasing the three components from three different suppliers or c) purchasing the three components from two randomly selected suppliers. Suppose that the suppliers are characterised by probabilities $x_{1}, x_{2}$ and $x_{3}$ of producing high-reliability components. Because the sequence $\{a\}=[3,0,0]$ majorizes the sequence $\{b\}=[1,1,1]$, the next inequality follows immediately from the Muirhead's inequality (2):

$$
(n-1) ! \times\left(x_{1}^{3}+x_{2}^{3}+x_{3}^{3}\right) \geq n ! \times x_{1} x_{2} x_{3}
$$

By dividing both sides of (6) to $n$ ! $(n=3)$, inequality (6) transforms into

$$
\frac{1}{3} x_{1}^{3}+\frac{1}{3} x_{2}^{3}+\frac{1}{3} x_{3}^{3} \geq x_{1} x_{2} x_{3}
$$

The left hand side of inequality (7) $\frac{1}{3} x_{1}^{3}+\frac{1}{3} x_{2}^{3}+\frac{1}{3} x_{3}^{3}$ is the probability of purchasing three high-reliability components from a randomly selected supplier. The right hand side of 
inequality (7) is the probability $x_{1} x_{2} x_{3}$ of purchasing three high-reliability components from three separate suppliers.

Since the sequence $\{a\}=[3,0,0]$ also majorizes the sequence $\{c\}=[2,1,0]$, the following inequality follows immediately from the Muirhead's inequality (2):

$$
(n-1) ! \times\left(x_{1}^{3}+x_{2}^{3}+x_{3}^{3}\right) \geq x_{1}^{2} x_{2}+x_{1}^{2} x_{3}+x_{2}^{2} x_{1}+x_{2}^{2} x_{3}+x_{3}^{2} x_{1}+x_{3}^{2} x_{2}
$$

Dividing both sides of ( 8$)$ by 3 ! $(n=3)$, gives

$$
\begin{aligned}
& (1 / 3) x_{1}^{3}+(1 / 3) x_{2}^{3}+(1 / 3) x_{3}^{3} \geq(1 / 3) \times\left[(1 / 2) x_{1}^{2} x_{2}+(1 / 2) x_{1}^{2} x_{3}\right]+ \\
& (1 / 3) \times\left[(1 / 2) x_{2}^{2} x_{1}+(1 / 2) x_{2}^{2} x_{3}\right]+(1 / 3) \times\left[(1 / 2) x_{3}^{2} x_{1}+(1 / 2) x_{3}^{2} x_{2}\right]
\end{aligned}
$$

The left hand side of inequality (9) gives the probability of purchasing three high-reliability components from a randomly selected single supplier. The right hand side of inequality (9) gives the probability of purchasing three high-reliability components from two randomly selected suppliers.

Suppose that the fractions of high-reliability components characterising the three suppliers are $x_{1}=0.9, x_{2}=0.75$ and $x_{2}=0.25$. The Monte-Carlo simulation based on 10 million trials resulted in probabilities $p_{1}=0.389$ and $p_{2}=0.26$ of purchasing three high-reliability components from a randomly selected single supplier and from two randomly selected suppliers, correspondingly. The left and right part of the inequality (9) yields $p_{1}=0.389$ and $p_{2}=0.26$ for the same probabilities, which illustrates the validity of inequality (9).

Finally, since the sequence $\{a\}=[2,1,0]$ majorizes the sequence $\{c\}=[1,1,1]$, the following inequality follows immediately from the Muirhead's inequality (2):

$$
x_{1}^{2} x_{2}+x_{1}^{2} x_{3}+x_{2}^{2} x_{1}+x_{2}^{2} x_{3}+x_{3}^{2} x_{1}+x_{3}^{2} x_{2} \geq n ! x_{1} x_{2} x_{3}
$$

Dividing both sides of (10) by 3 ! $(n=3)$, gives

$$
\begin{aligned}
& (1 / 3) \times\left[(1 / 2) x_{1}^{2} x_{2}+(1 / 2) x_{1}^{2} x_{3}\right]+(1 / 3) \times\left[(1 / 2) x_{2}^{2} x_{1}+(1 / 2) x_{2}^{2} x_{3}\right] \\
& +(1 / 3) \times\left[(1 / 2) x_{3}^{2} x_{1}+(1 / 2) x_{3}^{2} x_{2}\right] \geq x_{1} x_{2} x_{3}
\end{aligned}
$$

The left hand side of inequality (11) yields the probability of purchasing three high-reliability components from two randomly selected suppliers. The right hand side of inequality (11) yields the probability of purchasing three high-reliability components from three randomly selected suppliers.

For three suppliers are characterised by the probabilities $x_{1}=0.9, x_{2}=0.75$ and $x_{2}=0.25$ of selecting a high-reliability component, The Monte-Carlo simulation based on 10 million trials 
resulted in probabilities $p_{2}=0.26$ and $p_{3}=0.169$ of purchasing three high-reliability components from two randomly selected suppliers and from the three suppliers, correspondingly.

The left and right hand part of inequality (11) yield $p_{2}=0.26$ and $p_{3}=0.169$ for the same probabilities, which illustrates the validity of the inequality (11).

The same reasoning can be applied for any number of purchased components.

In summary, if no information is available about the components reliability characterising the separate suppliers, the best strategy is to purchase the components from a single supplier or from the smallest possible number of suppliers.

The same reasoning can be applied for any number of purchased components.

In summary, if no information is available about the components reliability characterising the separate suppliers, the best strategy is to purchase the components from a single supplier or from the smallest possible number of suppliers.

This section also demonstrates an important technique in using inequalities to improve reliability and reduce risk. It consists of giving appropriate meaning to the abstract variables in the derived inequalities, closely related to reliability improvement and risk reduction.

\section{Using inequalities for bounding deviations of reliability-critical parameters}

This section features a powerful method for improving reliability by limiting the deviations of reliability-critical parameters caused by errors associated with the design variables.

Estimating the absolute error associated with a particular quantity in the case where the average values of the design variables determining the quantity are known is a standard procedure from calculus, based on total differential. Indeed, consider an output quantity $z$ which is a smooth function $z=g\left(x_{1}, x_{2}, \ldots, x_{n}\right)$ of $n$ variables (parameters) $x_{1}, \ldots, x_{n}$, whose nominal (specified) values $x_{1 m}, \ldots, x_{n m}$ are known in advance. Suppose that the values $x_{1}, \ldots, x_{n}$ of the parameters vary around the specified nominal values $x_{1 m}, \ldots, x_{n m}$ with the small quantities $\Delta x_{1}, \ldots, \Delta x_{n}$. The absolute error $\Delta z$ associated with the output quantity $z$ is then determined from the total differential

$$
\Delta z=\left.\frac{\partial g}{\partial x_{1}}\right|_{x_{1}=x_{1 m}} \Delta x_{1}+\ldots+\left.\frac{\partial g}{\partial x_{n}}\right|_{x_{n}=x_{n m}} \Delta x_{n}
$$


which relates the absolute error $\Delta z$ of $z=g\left(x_{1}, \ldots, x_{n}\right)$ at the nominal values of the specified parameters $\left(x_{1 m}, \ldots, x_{n m}\right)$ to the errors $\Delta x_{1}, \ldots, \Delta x_{n}$ associated with the independent variables $x_{1}, \ldots, x_{n}$ at these nominal values.

In order to evaluate the absolute error $\Delta z$ from (12), the values of the partial derivatives at the point $\left(x_{1 m}, \ldots, x_{n m}\right)$ must be known.

Consider now the case where the nominal values $x_{1 m}, \ldots, x_{n m}$ of the independent variables $x_{1}, \ldots, x_{n}$ are unknown. The question of interest is to select the nominal values $x_{1 m}, \ldots, x_{n m}$ in such a way that the absolute error $\Delta z$ of the output quantity $z$ is minimised. If the absolute error is bounded in this way, it is guaranteed that the reliability-critical parameter cannot exceed a dangerous value and reliability cannot be compromised.

This important question can be answered by presenting the error $\Delta z$ of the output quantity as a function $f\left(x_{1}, \ldots, x_{n}\right)=0$ of $n$ variables and minimising this function under the constraint

$$
z=g\left(x_{1}, x_{2}, \ldots, x_{n}\right)
$$

which is effectively the function describing the output quantity.

According to the theory of multivariable optimisation (McCallum W.G., Hughes-Hallett D., Gleason A. M. et al., 2005), at the point of extremum, the equation:

$$
\operatorname{grad} f\left(x_{1}, \ldots, x_{n}\right)=\lambda \operatorname{grad} g\left(x_{1}, \ldots, x_{n}\right)
$$

and the equation

$$
g\left(x_{1}, \ldots, x_{n}\right)=0
$$

must be satisfied, where $\lambda$ is a constant of proportionality. These conditions are then used to derive the points at which the extremum is reached and also to evaluate the extremum. This approach will be illustrated by an application example from manufacturing.

Suppose that pieces of a particular material with volumes $V=7062 \mathrm{~mm}^{3}$ are delivered for a subsequent re-melting and processing. The pieces have a cylindrical shape with radius of the base $r$ and length $h$. The actual values of the dimensions $r$ and $h$ that guarantee the required volume of $V=7062 \mathrm{~mm}^{3}$ are not critical, as long as the volume $V$ does not deviate by more than $\Delta V=20 \mathrm{~mm}^{3}$ from the required value of $V=7062 \mathrm{~mm}^{3}$. If the volume $V$ deviates by more than $20 \mathrm{~mm}^{3}$ from the required value, the subsequent processing of the workpiece will result in a faulty component. Suppose that the absolute errors in the radius $r$ and the length $h$ of the cylindrical workpieces are $\Delta r=\Delta h=0.1 \mathrm{~mm}$. The question of interest is estimating the dimensions $r^{*}$ and $h^{*}$ with which the pieces must be cut so that the absolute error $\Delta V^{*}$ in 
the volume $V$ is the smallest possible. In other words, the dimensions $r^{*}$ and $h^{*}$ are sought that correspond to an absolute error $\Delta V^{*}$ such that the inequality:

$$
\Delta V \geq \Delta V^{*}
$$

holds, where $\Delta V$ is an absolute error that corresponds to dimensions $r$ and $h$, different from $r^{*}$ and $h^{*}$ but guaranteeing the required volume of $V=7062 \mathrm{~mm}^{3}$.

Since the volume of the cylindrical pieces is $V=\pi r^{2} h$, the absolute error associated with $V$ is given by $\Delta V=\frac{\partial V}{\partial r} \Delta r+\frac{\partial V}{\partial h} \Delta h$, or

$$
\Delta V=2 \pi r h \Delta r+\pi r^{2} \Delta h
$$

Substituting in (15) $\Delta r=\Delta h=0.1 \mathrm{~mm}$ yields

$$
\Delta V=0.2 \pi r h+0.1 \pi r^{2}
$$

Since the nominal values of $r$ and $h$ which guarantee the required volume $\mathrm{V}=7062 \mathrm{~mm}^{3}$ are unknown, to find the minimum absolute error $\Delta V$ in the required volume $V$, the function

$$
f(r, h) \equiv 0.2 \pi r h+0.1 \pi r^{2}
$$

must be minimised under the constraint

$$
g(r, h) \equiv \pi r^{2} h-7062=0
$$

The gradients of the functions $f(r, h)$ and $g(r, h)$ are:

$$
\operatorname{grad} f(r, h)=(0.2 \pi h+0.2 \pi r) \mathbf{i}+0.2 \pi r \mathbf{j}
$$

where $\mathbf{i}$ an $\mathbf{j}$ are the unit vectors along the $r$ and $h$ axis

$$
\operatorname{grad} g(r, h)=2 \pi r h \mathbf{i}+\pi r^{2} \mathbf{j}
$$

From $\operatorname{grad} f(r, h)=\lambda \operatorname{grad} g(r, h)$, the system of equations

$$
\begin{gathered}
0.2 \pi(h+r)=\lambda 2 \pi r h \\
0.2 \pi r=\lambda \pi r^{2}
\end{gathered}
$$

is obtained.

Dividing the left and the right hand parts of the equations results in $(h+r) / r=2 h / r$, from which, it follows that $h^{*}=r^{*}=\sqrt[3]{V / \pi}=\sqrt[3]{7062 / \pi}=13.1$ is a critical point. From the contour plots of the functions $f(r, h)$ and $g(r, h)$, it can be verified that the critical point is the minimum.

Consequently,

$$
\Delta V^{*}=0.2 \pi r h+0.1 \pi r^{2}=0.3 \pi r^{2}=0.3 \times \pi \times 13.1=12.35 .
$$

This means that the inequality 


$$
\Delta V \geq \Delta V^{*}
$$

has been proved regarding the absolute error $\Delta V$ associated with any other selected dimensions $(r, h)$ which guarantee the required volume $V$.

The absolute error is smaller than $20 \mathrm{~mm}^{3}$ therefore, the workpieces with dimensions $r=h=13.1 \mathrm{~mm}$ dimensions will not result in defective components. By selecting the nominal dimensions $h^{*}=r^{*}=13.1 \mathrm{~mm}$ the deviation of the volume $V$ due to errors in the dimensions $(r, h)$ has effectively been bounded. This guarantees that the volume $V$ cannot exceed a dangerous value and reliability cannot be compromised.

If for example, the dimensions $r=14.1$ and $h=11.31$ which deviate from the nominal values are selected, from

$$
\Delta V=2 \pi r h \Delta r+\pi r^{2} \Delta h=2 \pi \times 14.1 \times 11.31 \times 0.1+\pi \times 14.1^{2} \times 0.1=162.66 \mathrm{~mm}^{3}
$$

it can be seen that the error in $V$ significantly exceeds the maximum acceptable $20 \mathrm{~mm}^{3}$. If the dimensions $r=12.1, h=15.35$ which also deviate from the nominal values are selected, from

$$
\Delta V=2 \pi r h \Delta r+\pi r^{2} \Delta h=2 \pi \times 12.1 \times 15.35 \times 0.1+\pi \times 12.1^{2} \times 0.1=162.69 \mathrm{~mm}^{3}
$$

it can be seen that the error in $V$ again significantly exceeds the maximum acceptable of 20 $\mathrm{mm}^{3}$.

\section{Improving reliability and reducing risk by segmentation of loads and by chain-rule segmentation}

The underlying idea of the method of segmentation is to prevent failure modes and reduce the vulnerability to a single failure, by dividing an entity into a number of distinct parts (Todinov, 2019).

In this section, it is demonstrated that combining domain-specific knowledge from strength of materials and the domain-independent method of segmentation could achieve an increase in reliability by increasing the resistance to overstress failure.

\subsection{Reducing the risk of overstress failure by a segmentation of the external forces}

It is not at all obvious that segmenting external loading forces could achieve a significant reduction of the internal stresses in a loaded structure. In the cases where engineers have 
control over the design of the application points of external forces, a load segmentation can achieve a significant reduction of the internal stresses.

Consider the simply supported beam with length $l$ and uniform cross section in Figure 1, loaded with a concentrated force $P$. From the bending moment diagram, in section $x=l / 2$, the beam is subjected to a maximum moment $M_{\max , 1}=P l / 4$.

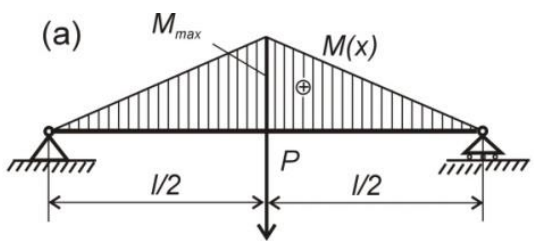

(b)

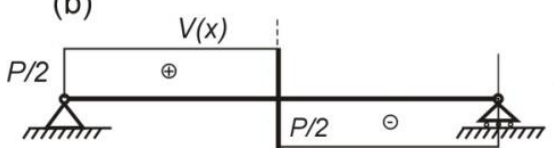

(c)

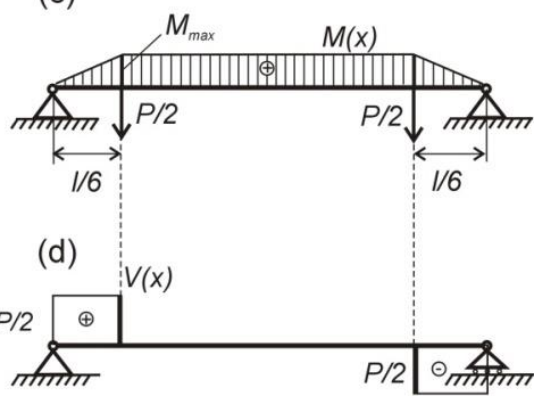

Figure 1. Reducing the risk of overstress failure of a beam by segmenting the external concentrated load $P$.

It is assumed that the design engineer has control over the application points of the external loads. Segmenting the concentrated load $P$ into two concentrated loads with magnitudes $P / 2$, applied at distances $l / 6$ from the supports, reduces the maximum bending moment which, in turn, reduces the internal tensile stresses from bending. Reducing the magnitudes of the internal tensile stresses increases the resistance to overstress failure and therefore improves reliability. A similar reliability improvement effect is also present if external concentrated moments, instead of concentrated forces are segmented.

The load segmentation also improves reliability in the case of a horizontal concentrated external load. This mechanism will be illustrated by the example of a statically indetermined loaded bar in Figure 2a, loaded with the external concentrated load $P$.

The stresses in the different sections of the bar in Figure 2a can be determined by using an extra compatibility of displacements equation. This is a standard technique documented, for example, in (Gere and Timoshenko, 1999). Neglecting the weight of the bar, as being much smaller compared to the magnitude of the concentrated force $P$, the stresses acting in the parts $A C$ and $C B$ of the bar are $\sigma_{A C}=\frac{P}{2 A}$ and $\sigma_{B C}=-\frac{P}{2 A}$, correspondingly (Figure $2 \mathrm{~b}$ ). 
(a)

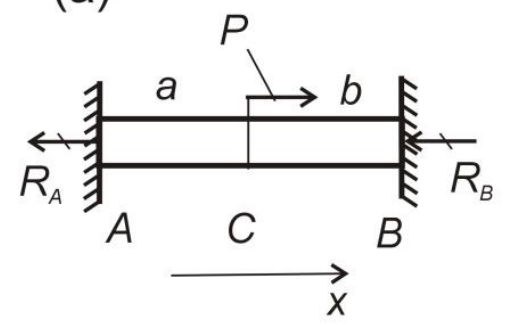

(b)

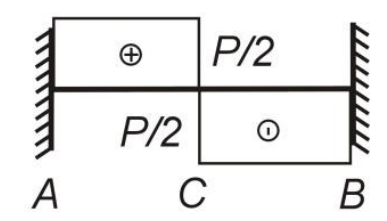

(c)

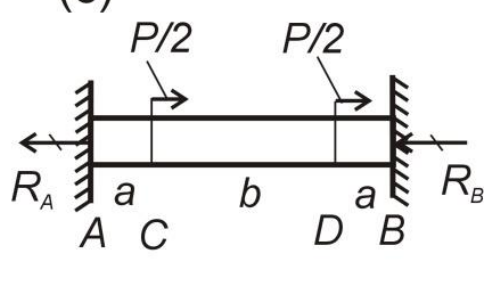

(d)

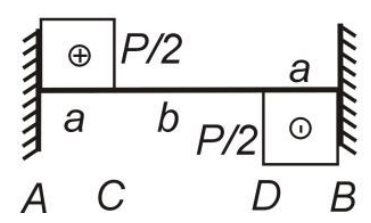

Figure 2. Reducing the overstress failure of a statically indeterminate bar by segmenting the external load

Now if the original concentrated load $P$ is segmented into two loads, each with magnitude $P / 2$, applied at the same distances $a$ from the supports (Figure 2c), the stresses acting in the parts $A C$ and $D B$ of the bar are still $\sigma_{A C}=\frac{P}{2 A}$ and $\sigma_{B C}=-\frac{P}{2 A}$, correspondingly, but the stress in any section of the part $C D$ with length ' $b$ ' is now zero (Figure 2d). This means that because of the reduced length where internal stresses are excited, the probability of failure due to buckling is reduced and the probability of failure due to a presence of a flaw in the material is also reduced.

Finally, consider the more complex truss structure in Figure 3a loaded by a $6 \mathrm{kN}$ external force. It is assumed again that the designer can control the points of application for the external load. The forces in the separate members have been calculated by the standard method of sections (Hibbeler, 2004) and are given in Table 1. The tensile forces are with plus sign while the compressive forces are with a negative sign.

As can be verified from Table 1, the loading with a single (non-segmented) force (Figure 3a) resulted in higher stresses in the members of the truss. Thus, the largest load for the truss with non-segmented load is $7.5 \mathrm{kN}$ (Table 1) while the largest load for the truss with segmented force is $5.25 \mathrm{kN}$ (Table 1). In addition, the magnitudes of the loads in the truss with segmented external load (Figure 3b) are more uniform compared with the truss with nonsegmented external load (Figure 3a). Indeed, the average value of the absolute values of the forces for the truss in Figure $3 \mathrm{a}$ is $4.91 \mathrm{kN}$ and the standard deviation of the absolute magnitudes of the forces is $2 \mathrm{kN}$. In contrast, the average value of the absolute values of the 
forces for the truss with segmented external load (Figure $3 \mathrm{~b}$ ) is $3.31 \mathrm{kN}$ and the standard deviation of the absolute magnitudes of the forces is $1.12 \mathrm{kN}$.

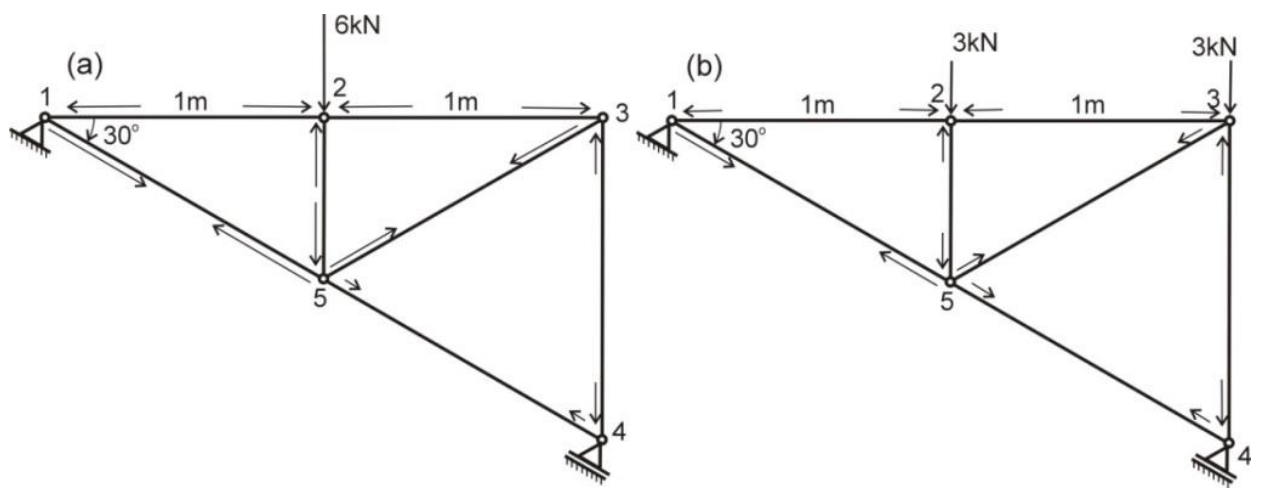

Figure 3. Reducing the overstress failure of a truss by segmenting the external load

Table 1 Forces calculated in the truss by the method of sections.

\begin{tabular}{|c|c|c|c|c|c|c|c|}
\hline Force $[\mathrm{kN}]$ & $\mathrm{S}_{12}$ & $\mathrm{~S}_{15}$ & $\mathrm{~S}_{23}$ & $\mathrm{~S}_{25}$ & $\mathrm{~S}_{34}$ & $\mathrm{~S}_{35}$ & $\mathrm{~S}_{45}$ \\
\hline Truss (a) & -5.196 & +7.5 & -5.196 & -6 & -3 & +6 & +1.5 \\
\hline Truss (b) & -2.59 & +5.25 & -2.59 & -3 & -4.5 & +3 & +2.25 \\
\hline
\end{tabular}

The presented simple solution for reducing the stresses in loaded structures based on segmentation has never been suggested in standard textbook on stress analysis and strength of components (Hearn 1985; Budynas, 1999; Gere and Timoshenko, 1999; Budynas and Nisbett 2015; Collins 2003; Norton, 2006). This shows that the lack of knowledge of the domainindependent method of segmentation made it invisible to the domain-specific experts that segmenting external loads can be used to reduce the internal stresses in a loaded structure and to reduce its risk of failure.

\subsection{Reducing the risk of computational errors by the method of chain-rule segmentation}

Segmentation is a universal domain-independent concept for risk reduction and can even be applied in the distant area related to reducing the risk of computational errors.

In this section, domain-specific knowledge from kinematic analysis of mechanisms and the domain-independent method of segmentation through the chain rule are combined to achieve a decrease in the risk of computational errors. 
The chain rule for differentiation of a function of a function is a well-known concept (Ellis and Gulick, 1991). The idea behind the concept of reducing errors by a segmentation through the chain rule is described next.

Suppose that a process output is a complex continuous function $y=y(x)$ of the input parameter $x$. Finding the derivative $\frac{d y}{d x}$ which describes the process output rate is often difficult and associated with a large likelihood of errors because of the complex function $y=y(x)$. The direct differentiation, if at all practicable, often leads to enormous, very complex expressions, during whose derivation the likelihood of making an error is very high. These difficulties disappear if segmentation through the chain rule is applied. The complex continuous function $y=y(x)$ is segmented into several simpler functions. Suppose that $y$ is expressed as a continuous function $y\left(u_{1}\right)$ of the parameter $u_{1}$; the parameter $u_{1}$ is expressed as a continuous function $u_{1}\left(u_{2}\right)$ of the parameter $u_{2}$ and so on, until a parameter $u_{n}$ is reached, which is expressed as a simple function $u_{n}(x)$ of $x$. As a result, $y=y(x)$ is effectively segmented to a nested composition of several functions:

$$
y=y\left(u_{1}\left(u_{2}\left(u_{3}\left(\ldots u_{n}(x)\right)\right)\right)\right.
$$

Applying the chain rule, for the derivative $d y / d x$, of the expression (25) gives

$$
\frac{d y}{d x}=\frac{d y}{d u_{1}} \times \frac{d u_{1}}{d u_{2}} \times \frac{d u_{2}}{d u_{3}} \times \ldots \times \frac{d u_{n}}{d x}
$$

Expression (26) is effectively a segmentation of the complex derivative $d y / d x$ into derivatives $d y / d u_{1}, d u_{1} / d u_{2}, d u_{n} / d x$ whose evaluation is relatively easy. Reducing the risk of errors comes from the circumstance that the evaluation of the separate derivatives $\frac{d u_{i}}{d u_{i+1}}$ is associated with a significantly smaller likelihood of error than the evaluation of the original derivative $\frac{d y}{d x}$. The complex task related to determining the rate $\frac{d y}{d x}$ has effectively been replaced by a number of sub-tasks with easy solutions. The solution of the original problem is assembled by multiplying the solutions of the partial problems, which is a straightforward operation. By making the differentiation of a complex expression easy through the chain-rule segmentation, the likelihood of errors is reduced significantly. 
The method of chain-rule segmentation remains the same if some of the parameters depend not on a single parameter but on two or more parameters. In this case, partial derivatives are used.

This chain-rule segmentation method will be illustrated with an example related to the specific domain "kinematic analysis of complex mechanisms". The mechanism in Fig.4, whose kinematics is to be modelled, incorporates a primary slider $B$ and a secondary slider $D$ connected to the primary slider by the rod $C D$, the pin joint $C$ and the link $C B$. The link $C B$ is welded firmly to the primary slider $B$ as shown in the figure and remains always perpendicular to the link $A T$. The crank $P A$ rotates in clockwise direction, with a uniform angular velocity of $\omega=2 \mathrm{rad} / \mathrm{s}$. The crank $P A$ subtends an angle $\varphi$ with the vertical axis and varies within the interval $[0,2 \pi]$ ( $0 \leq \varphi \leq 2 \pi)$. The values of the parameters fully specifying the mechanism are as follows:

$$
P A=r=0.35 m ; C B=s=0.65 m ; C D=m=0.75 m ; O P=p=0.60 m ; O B=d=0.85 m
$$

By using trigonometry, the coordinate $y=y(t)$ of point $D$ can be expressed as:

$$
y(t)=d+s \times \frac{p-r \cos (\omega t)}{\sqrt{[p-r \cos (\omega t)]^{2}+[d+r \sin (w t)]^{2}}}+\sqrt{m^{2}-\frac{s^{2}[d+r \sin (\omega t)]^{2}}{[p-r \cos (\omega t)]^{2}+[d+r \sin (\omega t)]^{2}}}
$$

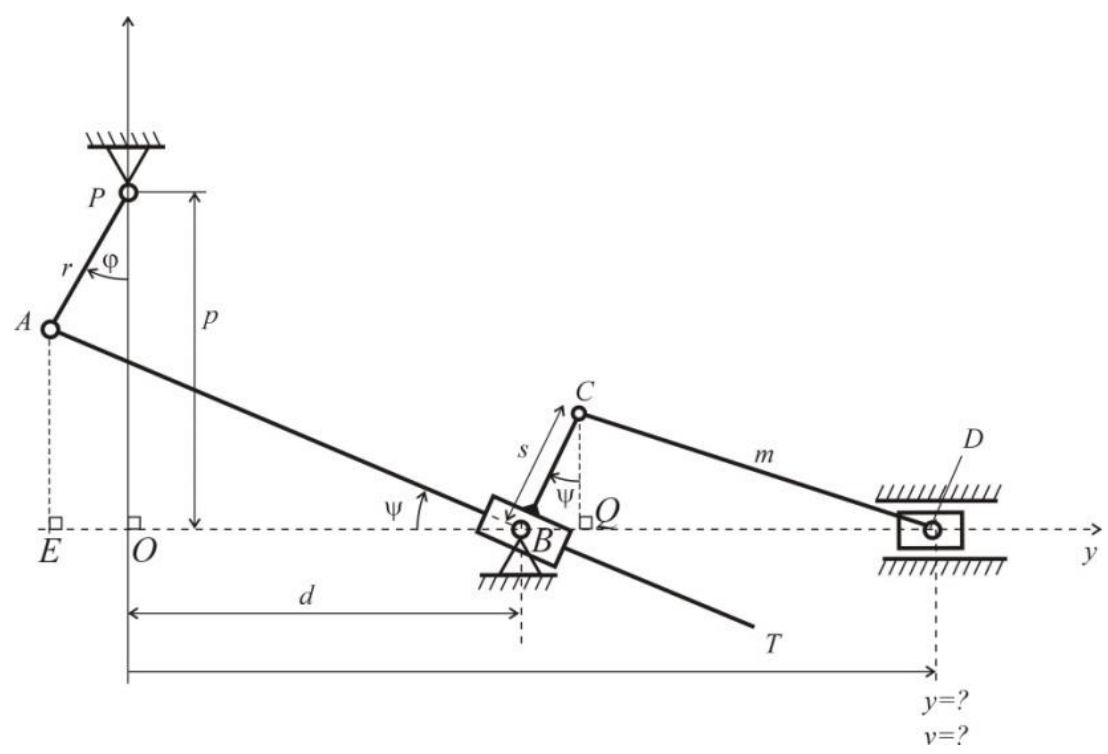

Figure 4. A mechanism whose kinematic analysis benefits from segmenting the problem through the application of the chain rule.

Differentiating expression (27) directly with respect to $t$ in order to determine the time dependence of the velocity of the slider $D(v(t)=d y(t) / d t)$ leads to a very complex expression, in whose derivation the likelihood of errors is very high.

However, $y(t)$ can be presented as a nested composition of three functions: 


$$
y(t)=y(\psi(\varphi(t)))
$$

each of which is easy to define:

$$
\begin{gathered}
y(\psi)=d+s \times \sin \psi+\sqrt{m^{2}-s^{2} \cos ^{2} \psi} \\
\psi(\varphi)=\tan ^{-1}\left(\frac{p-r \cos \varphi}{d+r \sin \varphi}\right)
\end{gathered}
$$

and $\varphi(t)=\omega t$.

Now the evaluation of the complex derivative $v(t)=d y(t) / d t$ is segmented by using the chain rule:

$$
d y(t) / d t=[d y / d \psi] \times[d \psi / d \varphi] \times[d \varphi / d t]
$$

Combining the derivatives by using the chain rule (26), results in

$$
d y / d t=\left\lfloor s \cos \psi+\frac{s^{2} \sin (2 \psi)}{2 \sqrt{m^{2}-s^{2} \cos ^{2} \psi}}\right\rfloor \times \frac{r^{2}+r d \sin \varphi-p r \cos \varphi}{r^{2}+d^{2}+p^{2}+2 d r \sin \varphi-2 p r \cos \varphi} \times \omega
$$

where the angle $\psi$ is given by the expression (29).

As a result, the chain-rule segmentation method avoids the difficult direct differentiation of expression (27) where the likelihood of making errors is significant. This is a powerful application of the method of segmentation in reducing the likelihood of computational errors while determining the rate of complex processes.

The graphs presenting the displacement and velocity of slider $D$ are given in Fig.5.

The correctness of the proposed chain-rule segmentation method (analytical expression (31)) in determining the velocity of the second slider, has been verified by using a direct (numerical) differentiation of expression (27) by discretising the angle $0 \leq \varphi \leq 2 \pi$ into small steps $h=0.001 \mathrm{rad}$. The value of the velocity for any time $t$ is given by

$$
v(t) \equiv d y / d t=(d y / d \varphi) \times(d \varphi / d t)
$$

Since $d y / d \varphi$ at an angle $\varphi_{i}$ can be approximated numerically by

$$
\left.(d y / d \varphi)\right|_{\varphi=\varphi_{i}} \approx \frac{y\left(\varphi_{i+1}\right)-y\left(\varphi_{i}\right)}{h},
$$

Consequently

$$
v_{i} \approx \frac{y\left(\varphi_{i+1}\right)-y\left(\varphi_{i}\right)}{h} \times \omega
$$




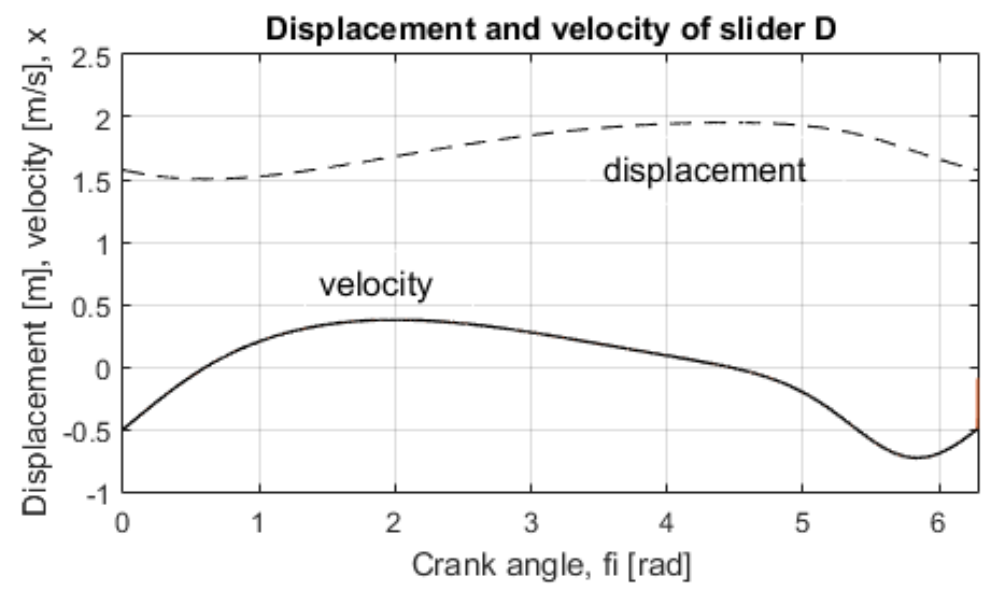

Figure 5. The velocity of slider $D$ obtained from the chain-rule segmentation method coincide with the velocity obtained from a direct numerical differentiation.

The results from the numerical differentiation for the velocity of slider $D$ coincide with the results for the velocity of slider $D$ calculated from the chain-rule segmentation method. The excellent correspondence of results obtained by two principally different methods validates the chain-rule segmentation method for reducing the risk of computational errors and demonstrates its validity.

To the best of our knowledge, the chain rule segmentation technique has not been discussed as a powerful analytic method for reducing the risk of computational errors in the analysis of complex mechanisms and machines (Uicker et al., 2017; Dicker et al, 2003; Sandor and Erdman, 1984). This example also demonstrates that the lack of knowledge of the method of segmentation made it invisible to experts that chain rule segmentation can be used with great success to reduce the risk of errors in complex calculations.

\section{CONCLUSIONS}

- A powerful domain-independent method for improving reliability and reducing risk based on algebraic inequalities has been introduced.

- A major advantage of algebraic inequalities has been demonstrated in ranking decision strategies in the absence of any knowledge related to the individual building elements.

If no information about the component reliability characterising the individual suppliers is available, purchasing components from a single supplier or from the smallest possible number of suppliers maximises the probability of a high-reliability assembly. 
- A major advantage of algebraic inequalities has been demonstrated in limiting the variation of a critical output parameter caused by variation of the design variables.

- The presented methods for risk reduction transcend mechanical engineering and work in many unrelated domains. The benefits from combining domain-independent methods with domain-specific knowledge to achieve risk reduction have been demonstrated in four unrelated application domains: decision-making, manufacturing, strength of components and kinematic analysis of mechanisms.

- A powerful chain-rule segmentation method for reducing the likelihood of computational errors during determining the rate of complex processes has been introduced and demonstrated in the area of kinematics analysis of complex mechanisms.

- Segmenting external loads can improve significantly the resistance to overstress failure by reducing the magnitudes of the internal stresses in loaded structures.

The presented research can be continued with: (i) new applications of algebraic inequalities for improving reliability and reducing risk; (ii) new applications demonstrating the benefits from combining domain-independent methods with specific knowledge in a particular domain to achieve effective risk reduction and (iii) developing new domain-independent methods for reliability improvement and risk reduction.

\section{REFERENCES}

Aven T., Risk assessment and risk management: review of recent advances on their foundation. European Journal of Operational Research; 2016; 253: 1-13.

Budynas R.G. Advanced strength and applied stress analysis, 2nd ed. New York: McGrawHill, 1999.

Budynas R.G., Nisbett J.K.. Shigley's Mechanical engineering design, 10th ed. New York: McGraw-Hill, 2015.

Childs P.R.N. Mechanical design engineering handbook. Amsterdam: Elsevier, 2014.

Cloud M., Byron C., Lebedev, L.P. Inequalities: with applications to engineering, New York: Springer-Verlag, 1998. 
Collins J.A. Mechanical design of machine elements and machines, New York: John Wiley \& Sons, Inc., 2003.

Dhillon B.S. Engineering systems reliability, safety, and maintenance. Boca Raton, New York: CRC Press, 2017.

Ebeling C.E. Reliability and maintainability engineering. Boston: McGraw-Hill, 1997.

Ellis R., D.Gulick, Calculus (one and several variables). New York: Harcourt Brace Jovanovich, Inc, 1991.

Engel A. Problem-solving strategies. New York: Springer, 1998.

French M. Conceptual design for engineers, 3rd ed. London: Springer-Verlag London Ltd, 1999.

Gere J. and Timoshenko S.P. Mechanics of Materials, 4th edn, Stanley Thornes Ltd, 1999.

Hardy, G., Littlewood J.E., Pólya, G. Inequalities. Cambridge Mathematical Library, Cambridge University Press, 1999.

Hearn E.J. Mechanics of materials, vol. 1 and 2, 2nd edition. Butterworth, 1985.

Hibbeler R.C. Statics and mechanics of materials, SI edition. Prentice Hall, 2004.

Kazarinoff N.D. Analytic Inequalities. New York: Dover Publications, Inc., 1961.

Lewis, E.E. Introduction to Reliability Engineering. New York:Wiley, 1996.

McCallum W.G., Hughes-Hallett D., Gleason A. M. et al. Calculus, multivariable, 4th edition, Wiley, 2005.

Modarres M., Kaminskiy M.P., Krivtsov V. Reliability engineering and risk analysis, a practical guide, 3rd ed. CRC Press, 2017.

Ning-Cong X., Hong-Zhong H., Yan-Feng L., Zhonglai W., Xiao-Ling Z., Non-probabilistic reliability sensitivity analysis of the model of structural systems with interval variables whose state of dependence is determined by constraints, Proc IMechE Part O: Journal of Risk and Reliability. 2013; 227(5): 491-498.

Norton R.L. Machine design, an integrated approach, 3rd ed. Pearson International edition, 2006.

O’Connor, P.D.T.. Practical Reliability Engineering, 4e. New York: Wiley, 2002.

Pahl G., W. Beitz, J. Feldhusen and K.H. Grote. Engineering design. Berlin: Springer, 2007.

Pachpatte B.G. Mathematical inequalities. North Holland Mathematical Library, vol.67, Amsterdam: Elsevier, 2005. 
Samuel A. and J. Weir. Introduction to engineering design: Modelling, synthesis and problem solving strategies, London: Elsevier, 1999.

Thompson G., Improving maintainability and reliability through design. London: Professional Engineering Publishing Ltd, (1999).

Rastegin A. Convexity inequalities for estimating generalized conditional entropies from below Kybernetika, 2012; 48 (2):242-253.

Steele J.M. The Cauchy-Schwarz master class: An introduction to the art of mathematical inequalities. New York: Cambridge University Press, 2004.

Todinov, M. Methods for reliability improvement and risk reduction. Wiley, 2019.

Todinov M.T.,. Limiting the probability of failure for components containing flaws, Computational Materials Science. 2005; 32: 156-166.

Uicker J.J.Jr, G.R.Pennock and J.E. Shigley. Theory of machines and mechanisms, 5rd ed., New York: Oxford University Press, 2017.

Sandor G.N. and Erdman A.G. Advanced mechanism design: analysis and synthesis vol.2, Englewood Cliffs NJ: Prentice-Hall Inc, 1984.

Dicker J.J., Pennock G.R., Shigley J.E. Theory of machines and mechanisms. Oxford: Oxford University Press, 2003. 